\title{
Micronutrients and weaning: an assessment of three books used for weaning guidance in Ireland
}

\author{
N. J. Canning, A. L. Kenny, E. M. Keaveney, A. E. Bennett, A. O’Connor and M. A. T. Flynn \\ The Food Safety Authority of Ireland, Dublin 1, Republic of Ireland
}

A shortfall of knowledge and a desire for specific information on weaning (defined as the transition from milk to solid foods) have been identified among parents in Ireland. From approximately the age of 6 months continued breast-feeding alongside the addition of nutritious solid foods is recommended to ensure adequate but not excessive intakes of essential micronutrients. Preliminary work by this group identified three self-help books used by parents for detailed weaning guidance. The aim of this study was to evaluate the micronutrient content of hypothetical 7-d food patterns developed from these books at various stages of weaning.

Weights for boys and girls at seven centiles $(0.4 \mathrm{th}, 2.5 \mathrm{th}, 25 \mathrm{th}, 50 \mathrm{th}, 75 \mathrm{th}, 91 \mathrm{st}$ and $99.6 \mathrm{th})$ at four ages corresponding to key stages in the weaning process $\left(5,7.5,10.5\right.$ and 12 months) ( $n 56$ infants) were derived using the UK-WHO growth charts ${ }^{(1)}$, and used to calculate fluid and nutrient goals. Seven-day food and breast milk intake patterns that complied with no added salt and minimal added fat and sugar were developed from the three books at the four weaning stages outlined above, for the fifty-six infants ( $n 1687$-d food and breast milk intake patterns). Nutrient intakes were compared with the Institute of Medicine's Dietary Reference Intakes ${ }^{(2,3)}$ for Fe, Zn, Ca, vitamin D, vitamin A and Na.

None of the 7-d intake patterns developed (containing oily fish and red meat) met the goals set for vitamin D (Fig. 1) or Fe (Fig. 2). Including a daily $5 \mu \mathrm{g}$ vitamin $\mathrm{D}_{3}$ supplement brought vitamin $\mathrm{D}$ intakes closer to the overall goal (Fig. 1). Intake patterns provided amounts of vitamin A (Fig. 3) and Zn (data not shown), which placed some infants above the tolerable upper level (UL) at all four weaning stages examined. Even at the earliest stage of weaning when intake was predominantly breast milk (5 months), infants who were at or above 75th centiles had intakes that exceeded the tolerable upper level for vitamin A (Fig. 3) and Zn (data not shown). Na intakes increased with age $(P<0.001)$ for food patterns based on all three books. At 12 months, 7-d intake patterns from Book-1, Book-2 and Book-3 provided 247, 151 and $219 \%$ (median intakes) of the adequacy goal for Na, respectively. All meal patterns provided Ca intakes at adequate intake (AI) levels (data not shown).

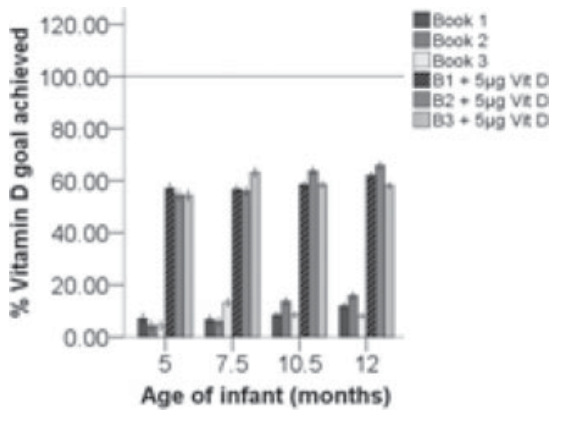

Fig. 1. \% Vitamin D goal met (EAR).

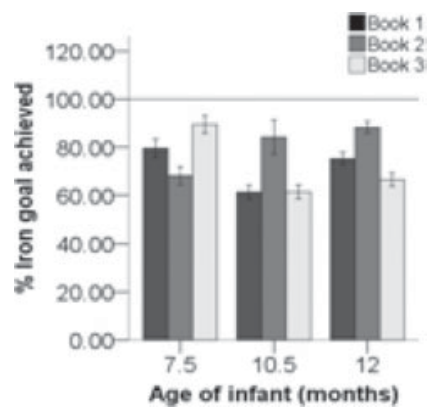

Fig. 2. \% Fe goal met (EAR).

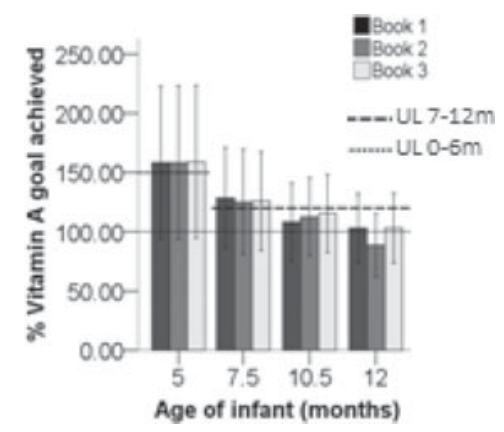

Fig. 3. $\%$ Vitamin A goal met (AI).

This study confirms that vitamin D supplementation of infants during the first year of life is necessary and that current recommendations of $5 \mu \mathrm{g}$ vitamin $\mathrm{D} / \mathrm{d}^{(4)}$ may need to be re-evaluated in light of more recent vitamin $\mathrm{D}$ intake goals ${ }^{(3)}$. In addition, the findings indicate that these supplements should not contain vitamin A. However, the finding that even predominantly breast-fed infants can exceed the UL for vitamin A and Zn questions the appropriateness of these UL. This study also highlighted the need for inclusion of highly bio-available sources of $\mathrm{Fe}$ in the infant diet. In conclusion, clear and practical public health information on weaning should be accessible to all parents.

1. UK-WHO (2010) Available at http://www.rcpch.ac.uk/Research/UK-WHO-Growth-Charts

2. Institute of Medicine (2006) Washington, DC: National Academies Press.

3. Institute of Medicine (2010) Washington, DC: National Academies Press.

4. Food Safety Authority of Ireland (2007) Dublin: Food Safety Authority of Ireland. Dublin. 